\title{
Treatment standards for spontaneous spinal epidural haematomas: management and main risk factors in era of anticoagulant/antiplatelet treatment
}

\author{
James F. Meschia ${ }^{\circ}$ \\ Department of Neurology, Mayo Clinic, Jacksonville, Florida, United States
}

A classical approach to providing insights into treatment of rare but serious disorders is provided by retrospective case series. In this issue of the Journal, Stetkarova et al. [1] report a series of 14 patients with spontaneous spinal epidural haematoma (SSEH) cared for at two University Hospitals in the Czech Republic over a decade. A total of $43 \%$ (6/14) were managed with decompressive spinal surgery, while the remaining patients were managed without surgery. A total of $71 \%(10 / 14)$ of the patients were taking either antiplatelet medication $(\mathrm{n}=2)$ or oral anticoagulant $(\mathrm{n}=10)$.

Spinal magnetic resonance imaging (MRI) is the test of choice for diagnosing SSEH. Ideally, patients suspected of SSEH should only receive care in hospitals that have the capacity to perform urgent spinal MRI studies at any time of the day or night. Not only diagnostically useful, spinal MRI allows for the evaluation of cord compression. When considering decompressive laminectomy, it is important to keep in mind that patients with cord compression from epidural haematoma can still have favourable postoperative outcomes despite intrinsic cord signal abnormalities [2].

The authors suggest that solely medical management should be reserved for patients with no, or mild, neurological deficit. Among the patients managed medically, six had presented with an American Spinal Injury Association Impairment Scale (AIS) Grade of D (i.e. at least half of key muscles below the neurological level having a motor grade of 3 or more) and one patient had an AIS Grade of E (normal motor and sensory function). However, one case managed medically presented with an AIS Grade of A (i.e. absence of all motor and sensory functions, including sacral roots, distal to the site of injury).

This 64-year-old woman recovered rapidly and completely, and this begs the question of how long patients should be observed for signs of recovery or deterioration before deciding whether or not the best course of action would be surgical decompression. The median time from symptom onset to decompression for the six patients who had surgery was 16.5 hours, with the longest being 28 hours. Presumably, if patients in the medical management-only group had had late deterioration of cord function, they would have ultimately had surgery.

Thus the series suggests that patients with SSEH and mild or no deficits ought to be observed with frequent neurological assessments in hospital for at least 24 hours before a decision is made as to whether the high-risk period for progressive cord compression with the need for surgery has passed.

The case series provides little information to guide physicians on the use of drugs or blood products to urgently reverse anticoagulation in patients who experience SSEH while taking an anticoagulant. Only one anticoagulated patient received an agent to reverse anticoagulation: a patient taking warfarin with an international normalised ratio (INR) of 5.2 received fresh frozen plasma. All other warfarin patients had either a therapeutic or a subtherapeutic INR when they bled.

The two patients taking direct oral anticoagulants (dabigatran and apixaban) had unremarkable tests of anticoagulant effect, though the article does not specify the timing of laboratory tests in relation to the onset of haemorrhage. For the much more common condition of intracerebral haemorrhage, the European Stroke Organisation Guidelines recommend the reversal of anticoagulation with warfarin with prothrombin complex concentrate and vitamin K; the reversal of dabigatran with idarucizumab; and the reversal of apixaban and rivaroxaban with andexanet alpha, although the quality of evidence for all of these recommendations ranged from very low to low [3].

Pending more robust evidence, it may be reasonable to use these ICH guidelines to guide medical management of SSEH,

Address for correspondence: James F. Meschia, M.D., Department of Neurology, Mayo Clinic, 4500 San Pablo Road, Jacksonville, Florida 32224, USA; e-mail: Meschia.james@mayo.edu

Received: 10.10.2021 Accepted: 11.10.2021 Early publication date: 12.10.2021

This article is available in open access under Creative Common Attribution-Non-Commercial-No Derivatives 4.0 International (CC BY-NC-ND 4.0) license, allowing to download articles and share them with others as long as they credit the authors and the publisher, but without permission to change them in any way or use them commercially. 
as in both conditions minor haematoma expansion can have disastrous consequences.

Retrospective surgical series involving one large hospital, or only a few, while useful, carry inherent limitations. Centres may be especially well-staffed with well-equipped and highly skilled surgeons, anaesthesiologists, theatre nurses, and others, making it challenging to generalise findings across different settings. The selection of patients for surgery or no surgery can vary over time and across different surgeons. Without pre-specified eligibility criteria, it can be challenging to translate findings to future cases. Case series often lack an assessment of standardised outcomes at regular intervals. Investigators should consider recontacting patients to assess long-term outcomes. Failure to collect long-term outcomes may give an overly pessimistic impression of prognosis. Recovery rates for traumatic spinal cord injury are better for patients followed for at least 12 months [4]. Presumably, recovery continues for patients with SSEH up to at least one year. In addition to AIS Grade, investigators should apply more generic validated functional outcome measures that have a demonstrated ability to detect differential efficacy in spinal surgery trials [5].

Physicians caring for patients with neurological disorders should be on the lookout for possible SSEH when patients present with a sudden, knife-in-the-back pain plus myelopathic signs and symptoms. Spinal MRI should be carried out immediately so that patients can receive timely treatment with decompressive laminectomy and reversal of anticoagulation, where appropriate.

\section{References}

1. Stetkarova I, Ehler E, Brabec K, et al. Spontaneous spinal epidural haematoma: management and main risk factors in era of anticoagulant/ /antiplatelet treatment. Neurol Neurochir Pol 2021; 55 (6): 574-581, doi: 10.5603/PJNNS.a2021.0066.

2. Baeesa S, Jarzem P, Mansi M, et al. Spontaneous spinal epidural hematoma: correlation of timing of surgical decompression and MRI findings with functional neurological outcome. World Neurosurg. 2019; 122: e241-e247, doi: 10.1016/j.wneu.2018.09.224, indexed in Pubmed: 30336292.

3. Christensen H, Cordonnier C, Kõrv J, et al. European Stroke Organisation Guideline on reversal of oral anticoagulants in acute intracerebral haemorrhage. Eur Stroke J. 2019; 4(4): 294-306, doi: 10.1177/2396987319849763, indexed in Pubmed: 31903428.

4. Khorasanizadeh M, Yousefifard M, Eskian M, et al. Neurological recovery following traumatic spinal cord injury: a systematic review and meta-analysis. J Neurosurg Spine. 2019; 30(5): 683-699, doi: 10.3171/2018.10.spine18802.

5. Ghogawala Z, Dziura J, Butler WE, et al. Laminectomy plus fusion versus laminectomy alone for lumbar spondylolisthesis. N Engl J Med. 2016; 374(15): 1424-1434, doi: 10.1056/NEJMoa1508788, indexed in Pubmed: 27074067. 